\title{
Comparison of the Multi-well Filtration ELISA, MPN and FISH Methods for Quantifying Ammonia Oxidizing bacteria in Activated Sludge
}

\author{
HAJIME IKUTA', MASATOSHI MATSUMURA', and YUHEI INAMORI ${ }^{2}$ \\ ${ }^{1}$ Institute of Applied Microbiology, Tsukuba University \\ / Tennodai 1-1-1, Tsukuba, Ibaraki 305-0006, Japan \\ ${ }^{2}$ National Institute for Environmental Studies / Onogawa16-2, Tsukuba, Ibaraki 305-0053, Japan
}

\begin{abstract}
MPN (most probable number) method has been used to quantify nitrifiers in activated sludge, and it was very useful for enumerating nitrifying activity. However, it takes about one month to get the results and requires a complicated operation. Consequently, in last decade, several immunological and molecular methods such as ELISA (EnzymeLinked Immunosorbent Assay) and FISH (Fluorescence in situ hybridization) methods have been developed to quantify nitrifiers rapidly. In this study, to enumerate ammonia oxidizing bacteria (AOB) population in activated sludge more rapidly, a new quantification method, multi-well filtration ELISA using a monoclonal antibody against Nitrosomonas europaea was developed. The threshold of the calibration curve using $N$. europaea with multi-well filtration ELISA method was about $4.0 \times 10^{5}$ cells $\cdot \mathrm{m} l^{-1}$, which was lower than that of normal ELISA. This means that the quantification range of AOB was extended. Furthermore, by using the multi-well filtration ELISA method, the bestowing time for biomass enumerating was decreased to 5 hours.

According to comparison of multi-well filtration ELISA, MPN and FISH methods, the property of multi-well filtration ELISA was more interrelated with that of MPN than that of FISH method when quantify $A O B$ in activated sludge under practical conditions. Furthermore, multi-well filtration ELISA took results the most quickly among them.
\end{abstract}

key words : ammonia oxidizing bacteria, nitrification, MPN, ELISA, FISH, activated sludge

\section{INTRODUCTION}

Nitrogen removal from industrial and domestic wastewater before discharging it into semi-closed water area such as lakes and marshes is essential to protect them from eutrophication. Since nitrifying bacteria play an important role in biological nitrogen removal, lack of microorganisms of this kind in wastewater treatment plants has become an extremely critical issue. Therefore, it is necessary to improve the reliability of nitrification in wastewater treatment, and first of all, to understand the ecology of nitrifying bacteria in a microbial community ${ }^{1)}$.

Biological nitrification, which consists of two reaction steps, the oxidation of ammonium $\left(\mathrm{NH}_{4}^{+}\right)$to nitrite $\left(\mathrm{NO}_{2}{ }^{-}\right)$and that of nitrite to nitrate $\left(\mathrm{NO}_{3}{ }^{-}\right)$, is principally performed by chemolitho-autotrophic ammonia oxidizing bacteria (AOB) and nitrite oxidizing bacteria (NOB). Though the nitrification process has been intensively studied for several decades, it is still difficult to realize stable nitrification performance in engineering systems. The main reason for this is the low growth rates of nitrifying bacteria. The specific growth rate of nitrifying bacteria is generally lower 
than that of heterophic bacteria which oxidize carbonaceous organic matter in wastewater treatment systems.

Cultivation-based methods such as MPN (most probable number) method have been used to detect nitrifiers. However, it takes about one month to get the results and requires a complicated operation. Furthermore, it has been increasingly recognized that the MPN technique is always selective and therefore can not yield sufficient documentation of the true community structure. Consequently, in last decade, several immunological and molecular methods such as ELISA (Enzyme-Linked Immunosorbent Assay) $^{2)}$ and FISH (Fluores-cence in situ hybridization) ${ }^{3)}$ methods have been developed to quantify nitrifiers rapidly without bias due to cultivation.

As a quantitative method for analysis of bacteria, the FISH method using Direct Cell Counts (DCC) is very useful for quantification of $\mathrm{AOB}^{4)}$. In this method, $\mathrm{AOB}$ is filtrated and mounted on membrane filter, and a $16 \mathrm{~S}$ rRNA-targeted specific probe for AOB is placed on it, and finally the hybridized AOB are directly counted under the fluorescent microscope. Because this method does not need pretreatment such as centrifuging, the accuracy of the assay is very high and the operation is performed more easily than the normal FISH method.

So far, we have investigated the ELISA method of assaying the nitrifiers, and have developed twelve hybridoma cell lines that produce monoclonal antibodies against Nitrosomonas europaea (IFO14298) as a representative $\mathrm{AOB}$, and these antibodies were composed of six IgM (named MAbne1-6) and six IgG $_{1}$ (named MAbne7-12). The calibration curve used to enumerate N.europaea was obtained by the sandwich ELISA method using the monoclonal antibodies ${ }^{5)}$. According to the result of calibration, the threshold of N.europaea quantification by sandwich ELISA was about $7.0 \times 10^{6} \mathrm{cells} \cdot \mathrm{m} l^{-1}$, and it took about 12 hours to obtain the result. However, when the amount of N.europaea is lower than $7.0 \times 10^{6} \mathrm{cells} \cdot \mathrm{m} l^{-1}$, centrifugation has to be carried out at first to concentrate them immediately before the analysis with sandwich ELISA. But when the nitrification activity is very low in wastewater treatment, the concentration of AOB in activated sludge will usually be lower than $7.0 \times 10^{6} \mathrm{cells} \cdot \mathrm{m} l^{-1}$. Accordingly, it is necessary to develop a method that can quantify AOB lower than 7.0 $\times 10^{6}$ cells $\cdot \mathrm{m} l^{-1}$ more easily and quickly.

With the MPN method, the quantification of AOB depends upon oxidation of ammonia, and the quantification by ELISA depends upon binding an enzyme labeled antibody to epitope such as protein or glycoprotein on the outer surface of target bacteria, and the FISH method depends upon the hybridization of fluorescent reagent labeled probe to $16 \mathrm{~S}$ rRNA of target bacteria. Therefore, the mechanisms of these methods are quite different, and it is necessary to investigate the property of these methods under a number of practical conditions.

The objectives of this study were (1) to develop a new quantification method, "multiwell filtration ELISA" to quantify AOB lower than $7.0 \times 10^{6}$ cells $\cdot \mathrm{m}^{-1}$ more easily and quickly, and (2) to compare the property of three quantification methods, MPN, multiwell filtration ELISA and FISH using DCC (DCC-FISH) to assay AOB in activated sludge.

\section{MATERIALS AND METHODS}

\section{Experimental apparatus and conditions}

The cultivation of activated sludge was carried out in a submerged membrane separation bioreactor that was developed as a model of the membrane separating activated sludge process. The schematic diagram of the experimental apparatus is shown in Fig. 1. It consisted of a $2.0 \mathrm{~L}$ aeration tank with membrane (pre size $0.4 \mu \mathrm{m}$ ) submerged in the water and a system to balance the flowrate change in influent. Nitrified wastewater at the aerobic reactor was separated from the microorganisms by membrane separation module.

In this experiment, two identical apparatuses were prepared. In both of these apparatus, the water temperature was set at $20^{\circ} \mathrm{C}$ at first, one was kept at $10^{\circ} \mathrm{C}$ from the 14 th day when the nitrification activity become stable (Run 1), and the other was not changed (Run 2). Hydraulic retention time (HRT) was adjusted to 12 hours. As seeding, 
activated sludge from the aerobic tank of a sewerage treatment facility was collected and inoculated. The dissolved oxygen and $\mathrm{pH}$ value were controlled at $2.0 \mathrm{mg} \cdot l^{-1}$ and 7.5 , respectively. Activated sludge was collected on the 0,4 th, 8th, 10th, 14th, 20th, 25th, 30 th, and 35th day after starting operation of the reactor. The concentration of ammonium nitrogen $\left(\mathrm{NH}_{4}-\mathrm{N}\right)$, nitrite nitrogen $\left(\mathrm{NO}_{2}-\mathrm{N}\right)$, nitrate nitrogen $\left(\mathrm{NO}_{3}-\mathrm{N}\right)$, and total nitrogen ( $\mathrm{T}-\mathrm{N})$ were determined by an automatic analyzer using a calorimetric method (BranLuebbe, TRAACS 800). The concentration of AOB was assayed by MPN, multi-well filtration ELISA and DCC-FISH method, respectively.

Monoclonal antibody The monoclonal antibody (MAbne6) against Nitrosomonas europaea (IFO14298) was utilized in this study, and it also cross reacts with Nitrosolobus multiformis (ATCC25196) as a representative $\mathrm{AOB}$ in the high percentage, while the cross reactivity against 9 heterotrophic bacteria living in activated sludge were at a very low level ${ }^{5)}$. The isotype of MAbne6 is IgG $_{1}$.

Multi-well filtration ELISA In this study, a 96 -well sterile micro titration plate designed with individual membranes (pore size ; $0.2 \mu \mathrm{m}$ ) sealed at the well bottom (multiscreen-GV ; MILIPORE co. Ltd.) was utilized (Fig.2).

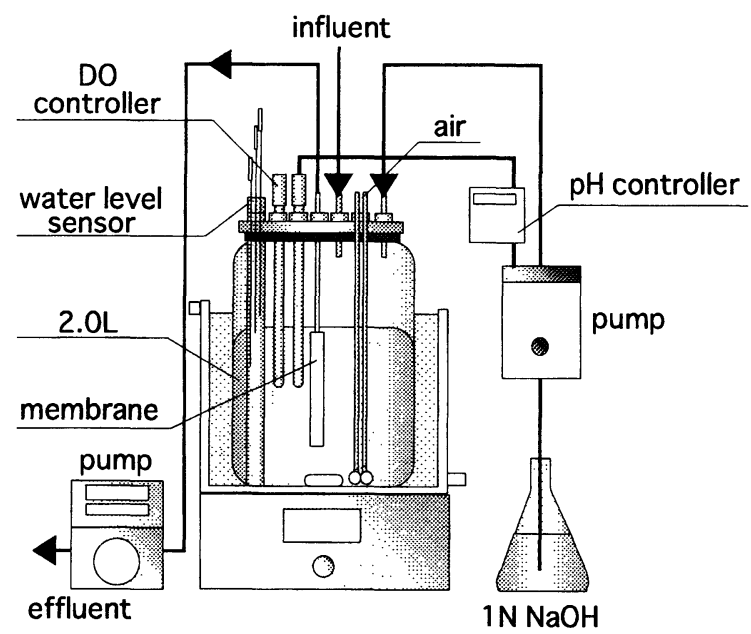

Fig. 1 Submerged membrane separation bioreactor
At first, collected samples containing AOB were diluted with phosphate-buffered saline (PBS) containing $0.05 \%$ Tween20 (T-PBS). After sonication for four minutes, sample solutions $(100 \mu l)$ were added to the wells, then the plate was mounted on a vacuum manifold and the filtrate was sucked out from the underdrain support structure at the bottom. When the AOB concentration of the sample was conjectured to be low, a larger amount of sample was added and filtrated. After blocking with $1 \mathrm{~g} / 100 \mathrm{ml}$ of Block Ace solution (Dainippon-pharmacy Corp.) at room temperature for 1 hour, the plate was sucked and washed three times with PBS. After washing, anti N.europaea monoclonal antibody solution (MAbne6, $100 \mu l$ ), which was used as the first antibody was added to every well and the plate was incubated at $37^{\circ} \mathrm{C}$ for 1 hour. After washing it three times with TPBS, goat-anti mouse IgG antibody labeled with alkali phosphatase $(50 \mu l$, ZYMED, $\times$ 1,000 dilution) was added to the wells as the second antibody and then the plate was incubated at $37{ }^{\circ} \mathrm{C}$ for 1 hour. After further washing (five times with PBS), substrate for the enzyme, p-nitrophenyl phosphate (1 $\mathrm{mg} / \mathrm{ml}$ diethanolamine buffer, $\mathrm{pH}$ 9.6), was added. After the plate was incubated at $37{ }^{\circ} \mathrm{C}$ for $30 \mathrm{~min}$, the reactants were retrieved to the wells of another micro titration plate, and the reactions were finally stopped by adding

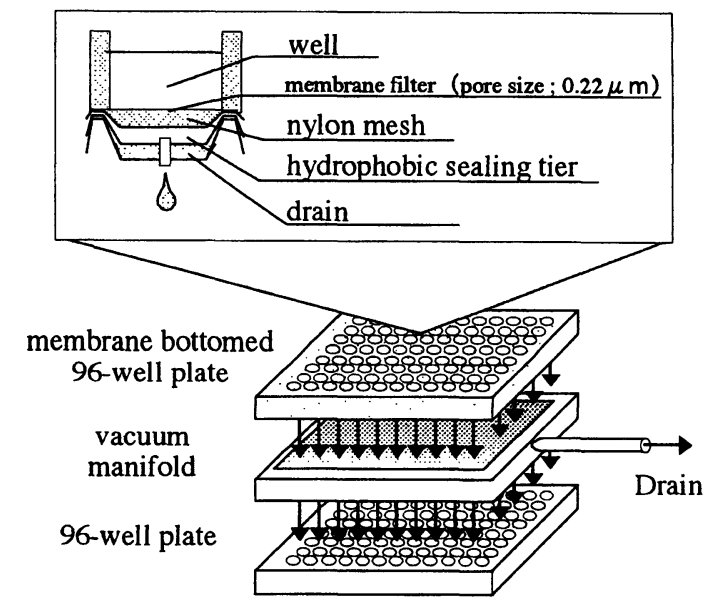

Fig. 2 Schematic diagram of the multi-well filtration ELISA. Assay was carried out on the membrane bottomed 96 -well plate 
$0.3 \mathrm{~N} \mathrm{NaOH}(50 \mu l)$. The absorbance of each well was measured by a microplate reader (BIO-RAD) at $405 \mathrm{~nm}$. The schematic of the multi-well filtration ELISA is shown in Figure 2. The calibration curve was developed in a pure culture of N.europaea to verify whether the multi-well filtration ELISA could be used as the rapid quantification method.

MPN method The MPN method used for enumerating AOB was carried out according to the method mentioned by Both et al. ${ }^{6)}$ Nitrification activity of the medium inoculated activated sludge was determined by the presence of nitrite and/or nitrate after 6 weeks of incubation at $30{ }^{\circ} \mathrm{C}$. The samples were dispersed by sonication for 4 minutes on ice immediately before assay. During the process of enumeration, each assay was carried out five times per one sample.

Fluorescence in situ hybridization targeting 16S rRNA The 16S rRNA-targeted oligonucleotides for ammonia oxidizing $\beta$ subclass Proteobacteria ${ }^{7}$, which was abbreviated as Nso190 was used as the specific probe for the FISH method. This probe was labeled with FITC (fluoresce in isothiocyanate) by TaKaRa Biotechnology (KALIAN) Co., Ltd..

A fixative solution of PBS buffer with $4 \%$ paraformaldehyde was prepared immediately prior to use. For in situ hybridization, samples were fixed by adding 3 volumes of fixative solution to 1 volume of cell suspension and kept at $4{ }^{\circ} \mathrm{C}$ for 2 hours ${ }^{8)}$. For quantitative analysis, the fixed cells were dispersed with $0.05 \%$ Tween 20 and after 4 minutes sonication. Then the cells were filtrated onto membrane filter surface (pore size ; $0.2 \mu \mathrm{m}$ ) and dehydrated by sequential filtration washes in 50, 80 and $98 \%$ ethanol (3 $\min$ each). Afterwards, a hybridization solution (25\% formamide, $0.9 \mathrm{M} \mathrm{NaCl}, 20 \mathrm{mM}$ Tris ( $\mathrm{pH} \quad 7.2$ ) and $0.01 \%$ sodium dodecyl sulfate (SDS)) containing $25 \mathrm{ng}$ of oligonucleotide probe was added. The membranes were incubated with hybridization solution for 2 hours at $46{ }^{\circ} \mathrm{C}$ in an isotonically equilibrated humid chamber. The hybridization solution was then removed by filtrating the membrane with washing solution $(25 \mathrm{mM} \mathrm{NaCl}, 20 \mathrm{mM}$ sodium phosphate ( $\mathrm{pH} 7.0), 0.01 \%$ SDS). Then, the membranes were kept in washing solution at $48{ }^{\circ} \mathrm{C}$ for $20 \mathrm{~min}$. To enumerate the total cells, 4',6-diamidino-2-phenylingole (DAPI) was added $\left(6.25 \mathrm{mg}\right.$-DAPI $\cdot l^{-1}, 1 \mathrm{M} \mathrm{NaCl}, \mathrm{pH}$ $7.2,5 \mathrm{~min})$. The filters were briefly rinsed with distilled water, and dried in the dark. After treatment with antifade reagent, the filter membrane was observed by a fluorescent microscope on excitation wavelength. Image processing and DCC were performed using a software package (Mac SCOPE Ver 2.32). During the DCC, cells in twelve fields were scanned and counted per membrane filter, and the average number was calculated but the maximum and the minimum number of cells fields were excluded and then DCC was given by the following equation :

$$
\begin{aligned}
& \text { DCC }\left(\text { cells } \cdot \mathrm{m}^{-1}\right)= \\
& \frac{\text { Cell count }(\text { cells }) \times \text { Fillter Area }\left(\mathrm{mm}^{2}\right) \times \text { Dilution Rate }}{\text { Fild Area for Counts }\left(\mathrm{mm}^{2}\right) \times \text { Sample Volume }(\mathrm{m} l)}
\end{aligned}
$$

\section{RESULTS AND DISCUSSION}

Calibration curve by multi-well filtration ELISA The calibration curve for enumeration of N.europaea obtained by means of multi-well filtration ELISA is shown in Figure 3. The result shows that multi-well filtration ELISA method can be used for estimating the biomass of N.europaea. The threshold of quantification of N.europaea by multi-well filtration ELISA was about $4.0 \times$ $10^{-5}$ cells $\cdot \mathrm{m}^{-1}$, and the upper limit was $5.0 \times$ $10^{7}$ cells $\cdot \mathrm{m} l^{-1}$. The samples which were more dense than $5.0 \times 10^{i}$ cells $\cdot \mathrm{ml}^{-1}$ can be quantified after pretreatment by diluting. This means that the quantification range of AOB was extended, as compared with sandwich ELISA. Furthermore, the bestowing time for biomass enumerating was reduced from 12 to 5 hours less than with sandwich ELISA.

In the sandwich ELISA, the assay was carried out on a normal 96 -well sterile micro titration plate which was completely sealed bottom, and AOB was fixed on the well bottom by binding with monoclonal antibody IgM specific for N.europaea ${ }^{51}$. On the other hand, in multi-well filtration ELISA, the 
plate designed with individual membranes sealed at the well bottom, and AOB was fixed on the well bottom by filtration. Therefore, it was assumed that the filtration fixed more $\mathrm{AOB}$ on the plate than binding with $\operatorname{IgM}$, and the quantification range of $\mathrm{AOB}$ was extended.

Nitrification Both of the membrane separation bioreactors achieved high performance wastewater treatment by the 10th day (Fig.4). $\mathrm{NH}_{4}-\mathrm{N}$ concentration of effluent continually decreased until the 10th day and finally reached less than $2.0 \mathrm{mg} \cdot l^{-1}$. After its

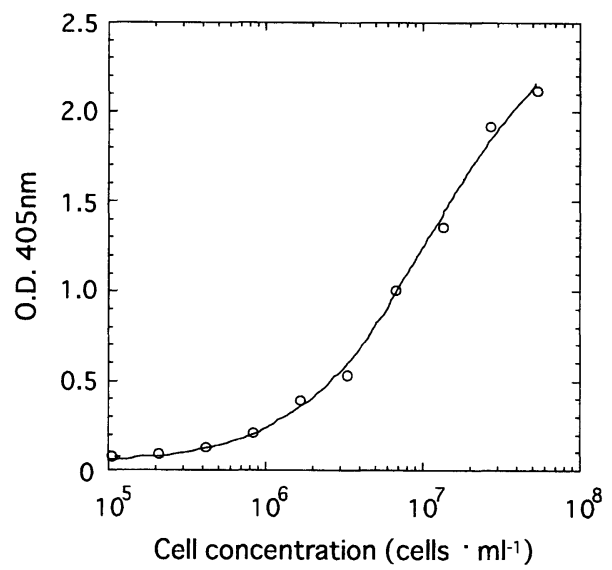

Fig. 3 Calibration curve for Nitrosomonas europaea (IFO14298). The assay obtained a lower detection limit of $4.0 \times 10^{5} \mathrm{cells} \cdot \mathrm{m}^{-1}$. $\mathrm{OD}_{405}$, optical density at $405 \mathrm{~nm}$.

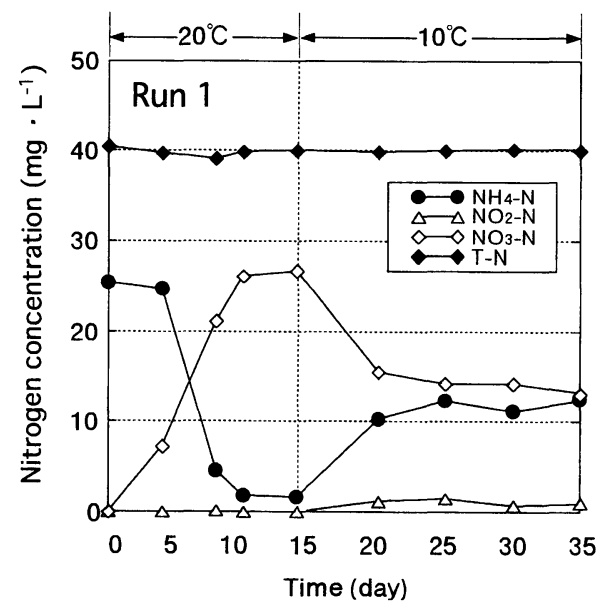

continually stable wastewater treatment performance capability was confirmed, the temperature of Run 1 was changed from 20 to $10{ }^{\circ} \mathrm{C}$ on the 14 th day. Thereby, it was observed that effluent $\mathrm{NH}_{4}-\mathrm{N}$ concentration increased over $10 \mathrm{mg} \cdot l^{-1}$ until the 20th day and did not decrease after the 20th day. Run 2 maintained a high rate of nitrification after the 14th day.

Comparison of three methods The results of $\mathrm{AOB}$ concentration at start up of the operation were $1.0 \times 10^{6} \mathrm{cells} \cdot \mathrm{m} l^{-1}, 2.6 \times$ $10^{6}$ cells $\cdot \mathrm{m} l^{-1}$, and $1.2 \times 10^{6}$ cells $\cdot \mathrm{m} l^{-1}$ by MPN, multi-well filtration ELISA, and DCCFISH methods respectively (Figs. 5), and the results were in the order: multi-well filtration ELISA $>$ DCC-FISH $>$ and MPN but the difference among them was little. In both Run 1 and 2, the concentration of $\mathrm{AOB}$ by MPN and multi-well filtration ELISA were increased immediately from start up, although that by DCC-FISH did not increase until the 8th day. The AOB concentrations on the 14 th day were $8.6 \times 10^{7}, 5.6 \times 10^{7}$, and 6.1 $\times 10^{6}$ cells $\cdot \mathrm{m}^{-1}$ by MPN, multi-well filtration ELISA, and DCC-FISH methods respectively in Run 1. On the other hand, in Run 2, they were $6.5 \times 10^{7}, 5.3 \times 10^{7}$, and $5.4 \times 10^{6}$ cells. $\mathrm{m} l^{-1}$ by MPN, multi-well filtration ELISA, multi-well filtration ELISA, and DCC-FISH methods respectively. The order of $\mathrm{AOB}$ concentration by the three methods, which was same in both experimental apparatus

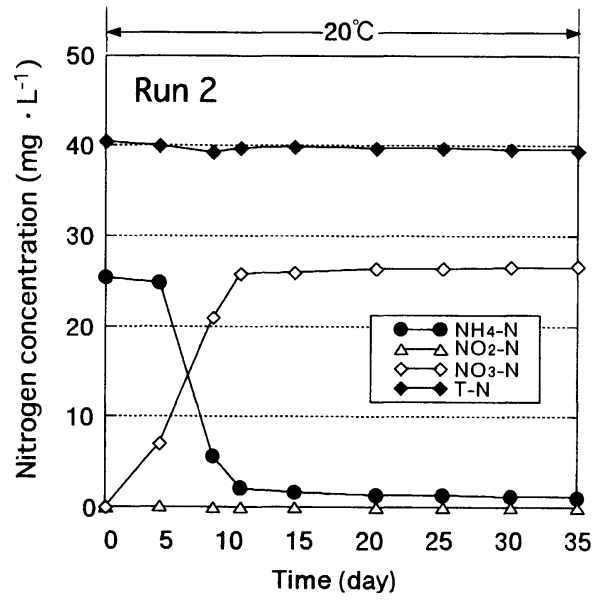

Fig. 4 Time course of $\mathrm{NH}_{4}-\mathrm{N}, \mathrm{NO}_{2}-\mathrm{N}, \mathrm{NO}_{3}-\mathrm{N}$, and $\mathrm{T}-\mathrm{N}$ concentrations in effluent. In Run 1, the water remparature was changed from 20 to $10{ }^{\circ} \mathrm{C}$ on the 14 th day, and that of Run 2 was kept constant. 
Run 1 and 2, was MPN > multi-well filtration ELISA $>$ and DCC-FISH, and the concentrations by MPN and multi-well filtration ELISA were ten times higher than that by DCC-FISH. The water temperature of Run 1 was changed from 20 to $10{ }^{\circ} \mathrm{C}$ on the 14th day, and the increase of the AOB concentrations by MPN and multi-well filtration ELISA stopped for a while, and eventually decreased. However, the AOB concentration by DCC-FISH increased a little and did not decrease again. In Run 2, they did not decrease at all until the 35 th day with the three methods. At this time, from the 20 th to the 35 th day in Run 1 , the AOB decreased by 98.5 and $80.1 \%$ using MPN and multi-well filtration ELISA respectively, and the value of decay rate constants $\left(K_{d}\right)$ for AOB were 0.13 and 0.06 day $^{-1}$, which were given by $\mathrm{K}_{\mathrm{d}}=(1 / \mathrm{T}) \times \log \mathrm{N}_{0} / \mathrm{N}_{\mathrm{t}}$ (Fig. 6).

From this result, it was found that the AOB concentration by the MPN method was a little higher than that of multi-well filtration ELISA when the activity of nitrification was high, but the difference between them was very little and the property of the quantification by multi-well filtration ELISA interrelated a lot with that of the MPN method in activated sludge. On the other hand, as a whole, although the initial concentrations were closed to each other, $\mathrm{AOB}$ concentration obtained by the DCC-FISH method was lower than the other methods. From this result, it was assumed that the main AOB grew in the reactor couldn't be detected by the Nso190 probe. Furthermore, the AOB concentrations by DCC-FISH were not decreased after the water temperature was changed from 20 to $10{ }^{\circ} \mathrm{C}$ in Run 1 , although the concentration was lower than that in Run 2 at that time. From the result of this experiment, it was assumed that the observation of $\mathrm{AOB}$ concentration decline caused by temperature change is very difficult to be detected by DCCFISH method.

The AOB concentrations by three methods in Run 1 and 2 were summarized, and the quantified AOB concentrations by the MPN method were compared with those by the multi-well filtration ELISA and DCC-FISH as shown in Figure 7. As a result, when AOB concentration was lower than $2.5 \times 10^{7}$ cells . $\mathrm{m} l^{-1}$, the result by multi-well filtration ELISA was little higher than that of MPN. After AOB concentration reached around 2.5 $\times 10^{7}$ cells $\cdot \mathrm{m} l^{-1}$, result by multi-well filtration ELISA shifted to be lower than that of MPN. The higher the concentration, the more the difference. According to these results, MPN method seemed to be the best among these three methods mentioned. But multi-well filtration ELISA method could also be regarded to be similar in sensitivity company with MPN, because difference, as a whole, was less than ten times. The multi-well
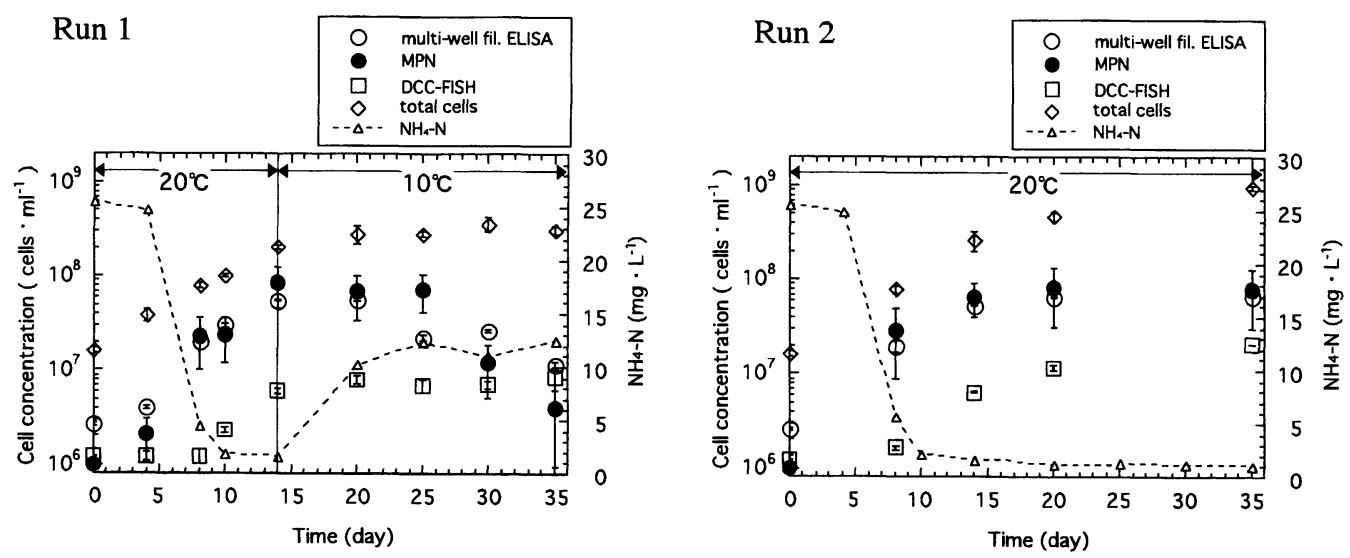

Fig. 5 Time courses of $A O B$ concentrations which were quantified with the multi-well filtration ELISA, MPN, and FISH using DCC methods. In Run 1, the water temperature was changed from 20 to $10{ }^{\circ} \mathrm{C}$ on the 14 th day, and that of Run 2 was kept constant. 


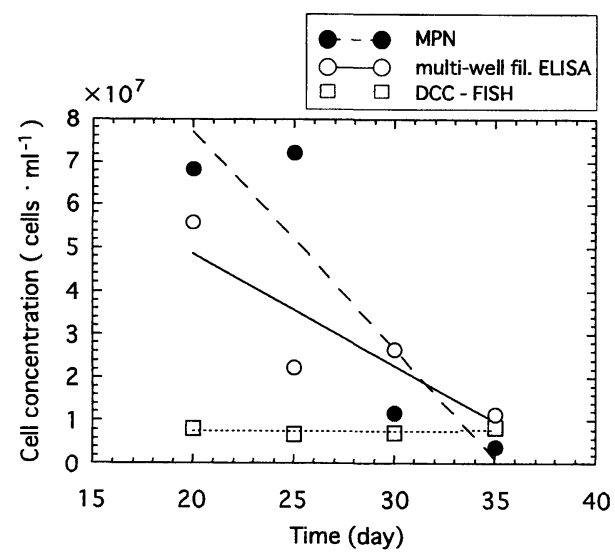

Fig. 6 Decrease of $A O B$ concentration from the 20th to 35 th day at $10{ }^{\circ} \mathrm{C}$ in Run 1 . The value of decay rate constants $\left(K_{d}\right)$ were 0.13 day $^{-1}$ with MPN and 0.06 day $^{-1}$ with multi-well filtration ELISA.

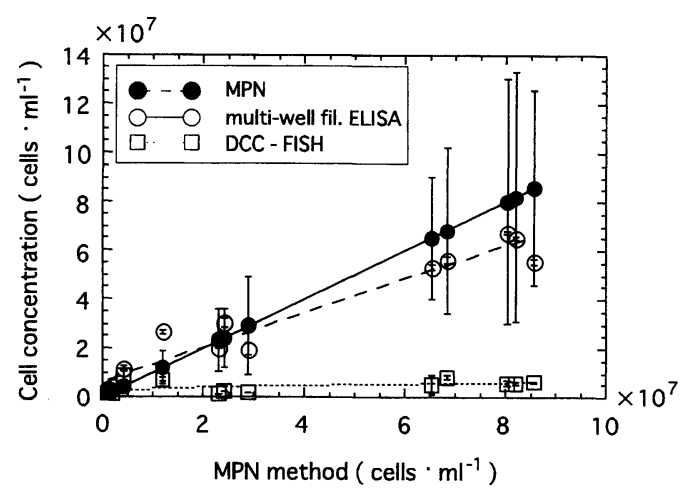

Fig. 7 The comparison of quantified $A O B$ concentrations obtained by the MPN method with those obtained by the multi-well filtration ELISA and DCC-FISH methods.

filtration ELISA method, then, had the advantage that it takes only 5 hours to determine AOB concentration, and the standard deviation of the result was very low, as compared with MPN method.

\section{SUMMARY}

In this study, multi-well filtration ELISA was developed to enumerate the biomass of AOB more easily and quickly. Furthermore, comparing the property of three quantification methods - MPN, multi-well filtration ELISA, and FISH using DCC - to detect AOB from activated sludge obtained the following results.

1) The threshold of the calibration curve for N.europaea by multi-well filtration ELISA was about $4.0 \times 10^{5}$ cells $\cdot \mathrm{ml}^{-1}$, which was lower than that of sandwich ELISA. Furthermore, comparing sandwich ELISA with multi-well filtration ELISA method revealed that the bestowing time for biomass enumerating was decreased from 12 to 5 hours.

2) The AOB concentrations by multi-well filtration ELISA were higher than that of FISH using DCC method, and they were closed to that of MPN.

3) The property of multi-well filtration ELISA under practical conditions such as temperature change was interrelated with that of MPN method when quantifying AOB in activated sludge.

4) It was considered that multi-well filtration ELISA was the most useful method for quantifying $\mathrm{AOB}$ in activated sludge among the three methods in this study.

\section{REFERENCES}

1) Antoniou P., Hamilton J., Koopman B., Jain R., Holloway B., Lyberatos G., and Svoronos S.A.: Effect of temperature and $\mathrm{pH}$ on the effective maximum specific growth rate of nitrifying bacteria. Wat. Res., 24 (1), 97-101 (1990).

2 ) N.Saraswat, J. E. Alleman, and T. J. Smith.: Enzyme Immunoassay Detection of Nitrosomonas europaea.Appl. Environ. Microbiol., 60, 1969-1973 (1994).

3 ) Amann R. I., Krumholz, L., and Stahl, D. A.: Fluorescent-oligonucleotide probing of whole cells for determinative, phylogenetic, and environmental studies in microbiology. J. Bacteriol., 172, 762770 (1990).

4 ) Araki, N., Ohashi, A., Harada, H., and Hachdar, I.: Direct Cell Counting and Observation of Spacial Distribution of Nitrifiers in Aerobic Biofilms by FISH (fluorescent insitu hybridiztion) (In Japanese). J. Jap. Soc.Water Environ., 22, 152-159 (1999).

5 ) Ikuta, H., Noda, N., Ebie Y., Hirata, A., Tsuneda, S., Matsumura, M., and Inamori, Y.: The rapid quantification and 
detection of Nitrifying bacteria by using monoclonal antibody method. Water Science and Technology., 42, 1-7 (2000).

6 ) Both G. J., Gerards S., and Laanbroek H. J.: The occurrence of chemolithoautotrophic nitrifiers in waste-saturated grassland soils. Microb. Ecol., 23, 15-26 (1992).

7 ) Mobarry, B. K., M. Wagner, V. Urbain, B. E. Rittmann, and D. A. Stahl.: Phylogenetic probes for analyzing abundance and spatial organization of nitrifying bacteria. Appl. Environ.
Microbiol., 62, 2156-2162 (1996).

8 ) Amann R. I.: In situ identification of micro-organisms by whole cell hybridization with rRNA-targeted nucleic acid probes, chapter 3.3.6, In: Molecular microbial ecology manual, A. D. I. Akkermans, J. D. Van Elsas, and F. J. De Bruijn (ed), Kluwer Academic Publishers, Dordrecht, The Netherlands (1995).

(Submitted 2001. 10. 15)

(Accepted 2002. 1. 30) 